\title{
Uncertainties about interpretation of ventricular stimulation studies in patients with Wolff-Parkinson-White syndrome
}

\author{
KILLIAN ROBINSON, DENNIS M KRIKLER \\ From the Division of Cardiovascular Disease, Royal Postgraduate Medical School, London
}

Arrhythmia is common and important in the Wolff-Parkinson-White syndrome, and indeed many would agree that both pre-excitation and supraventricular arrhythmia are essential features of the classical expression of this syndrome. Reentrant atrioventricular tachycardia is the most commonly. encountered arrhythmia in the syndrome, but atrial fibrillation and to a lesser extent flutter are seen in $10-32 \%$ of patients. ${ }^{1}$ Independent, coincidental arrhythmias, such as intranodal reciprocating tachycardia, are unusual. ${ }^{2}$ There is of course the fear of ventricular fibrillation ${ }^{34}$; and a small risk of sudden death has been reported. ${ }^{3-5}$

In this issue of the British Heart Journal (p. 89), Brembilla-Perrot and her colleagues examine the occurrence of ventricular tachycardia in the WolffParkinson-White syndrome. They compared the electrophysiological findings in $\mathbf{4 6}$ patients with preexcitation with those in 36 controls in whom there was no evidence of pre-excitation. Programmed stimulation induced ventricular tachycardia in 37\% of patients with pre-excitation and in $3 \%$ of the controls. The problem in the Wolff-Parkinson-White syndrome is not, however, ventricular tachycardia but rather ventricular fibrillation.

Clinically significant spontaneous ventricular tachycardia is rare in the pre-excitation syndrome: only one case was noted in each of two series of 139 and 163 patients $^{67}$ : and another review of reported cases of pre-excitation also found that this arrhythmia was uncommon. ${ }^{8}$ When spontaneous ventricular tachycardia occurs it seems to be associated with underlying heart disease. ${ }^{9}$ Furthermore, when

Requests for reprints to Dr Dennis M Krikler, Division of Cardiovascular Disease, Hammersmith Hospital, 150 Ducane Road, London W12 0HS. ventricular fibrillation complicates the WolffParkinson-White syndrome it is usually preceded by atrial fibrillation and not by ventricular tachycardia. Non-sustained multiform ventricular tachycardia is often observed at electrophysiological study and may be induced in normal individuals if the stimulation protocol is sufficiently aggressive. ${ }^{10} \mathrm{~W}$ ith the protocol used in the current study, and in that of Milstein $e t a l,{ }^{11}$ however, the expected frequency of induced ventricular tachycardia would be low, perhaps $0.7 \%{ }^{12}$ or $3 \% .^{13}$

Nevertheless, there are now two studies, the present one and that of Milstein et al, ${ }^{11}{ }^{1}$ which show that inducible ventricular tachycardia is more common in patients with the Wolff-Parkinson-White syndrome than in control groups. The question whether this feature is a further non-specific, but sensitive, indicator of the risk of ventricular fibrillation will only be resolved by long term follow up and knowledge of other possible risk factors, such as a history of both reciprocating tachycardia and atrial fibrillation with short maximally pre-excited RR intervals. ${ }^{4}$ On its own, however, the importance of inducible ventricular tachycardia is doubtful because the frequency of sudden death in the preexcitation syndrome is not even remotely near $37 \%$ : in clinical experience it is rare, and it was not encountered in one review. ${ }^{14}$

How do these findings affect the management of patients with the Wolff-Parkinson-White syndrome? The answer is hardly at all. From a clinical standpoint, decisions will continue to be based either on symptoms or on the presumed risk of ventricular fibrillation as assessed by the anterograde conduction properties of the accessory pathway. ${ }^{15}$ From the epidemiological point of view, as various forms of treatment, including surgical ablation of 
the accessory pathway, reduce the number of patients with the Wolff-Parkinson-White syndrome considered to be at high risk, it will become impossible to evaluate any dangers of susceptibility to inducible ventricular tachycardia and its relation to other high risk factors for sudden death. This may be for the best as, whatever the shortcomings of assessing risk from the conduction properties of the accessory pathway, there will be endless problems, both medical and socioeconomic, if too much weight is placed on non-specific ventricular responses to programmed stimulation. Indeed, in a recently published consensus statement there was no agreement about the value of programmed stimulation in the identification of ventricular tachycardia (save in specific circumstances), and in the Wolff-Parkinson-White syndrome it was proposed that very rigid criteria, including drug-refractory arrhythmia, must be fulfilled before routine study is warranted. ${ }^{16}$ Longevity in the WolffParkinson-White syndrome is by no means rare ${ }^{17}$ and one should be very careful before deducing high risk from the results of invasive studies, in the face of clinical experience.

This having been said, the results presented by Brembilla-Perrot and her colleagues will be studied with interest and taken into account by those performing electrophysiological studies in patients with the Wolff-Parkinson-White syndrome. It should not take long to assess whether ventricular stimulation of this sort is relevant to the Wolff-Parkinson-White syndrome; this will give us more confidence in the management of our patients with this syndrome. If the clinical relevance of what Brembilla-Perrot et al say turns out to be highly limited, it will provide further reassurance about the management of the vast majority of patients with this disorder.

\section{References}

1 Campbell RWF, Smith RA, Gallagher JJ, Pritchett ELC, Wallace AG. Atrial fibrillation in the pre-excitation syndrome. Am J Cardiol 1977; 40:514-20.

2 Spurrell RAJ, Krikler D, Sowton E. Two or more intra AV nodal pathways in association with either a James or Kent extranodal bypass in 3 patients with paroxysmal supraventricular tachycardia. Br Heart $J$ 1973;35:113-22.

3 Dreifus LS, Haïat R, Watanabe Y, Arriaga J, Reitman N. Ventricular fibrillation: a possible mechanism of sudden death in patients with the Wolff-Parkinson-White syndrome. Circulation 1971;43:520-7.
4 Klein GJ, Bashore TM, Sellers TD, Prichett ELC, Smith WM, Gallagher JJ. Ventricular fibrillation in the Wolff-Parkinson-White syndrome. $N$ Engl J Med 1979;301:1080-5.

5 Brembilla-Perrot B, Aliot E, Louis P, et al. Devenir de 195 patients atteints de syndrome de Wolff-Parkinson-White. Arch Mal Coeur 1987; 80:271-7.

6 Wellens HJJ. The electrophysiological properties of the accessory pathway in the Wolff-Parkinson-White syndrome. In: Wellens HJJ, Lie KI, Janse MJ, eds. The conduction system of the heart. Philadelphia: Lea and Febiger, 1976:567-87.

7 Benditt DG, Pritchett ELC, Gallagher JJ. Spectrum of regular tachycardia with wide QRS complexes in patients with accessory atrioventricular pathways. Am J Cardiol 1978;42:828-38.

8 Reddy CP, Sartini JC, Kuo CS. Paroxysmal ventricular tachycardia in Wolff-Parkinson-White syndrome: case report and review of the literature. J Electrocardiol 1982;15:403-10.

9 Krikler DM, Davies MJ, Rowland E, Goodwin JF, Evans RC, Shaw DB. Sudden death in hypertrophic cardiomyopathy: associated accessory atrioventricular pathways. Br Heart $J$ 1980;43:245-51.

10 Brugada P, Green M, Abdolleh H, Wellens HJJ. Significance of ventricular arrhythmias initiated by programmed ventricular stimulation: the importance of the type of ventricular arrhythmias induced and the number of premature stimuli required. Circulation 1984;69:87-92.

11 Milstein S, Sharma AD, Klein GJ. Non clinical ventricular tachycardia in the Wolff-Parkinson-White syndrome. PACE 1985;8:178-83.

12 Vandepol CJ, Farshidi A, Spielman SR, Greenspan AM, Horowitz LN, Josephson ME. Incidence and clinical significance of induced ventricular tachycardia. Am J Cardiol 1980;45: 725-31.

13 Livelli FD, Bigger JT, Reiffel JA, et al. Response to programmed ventricular stimulation: sensitivity, specificity and relation to heart disease. Am J Cardiol 1982;50:452-8.

14 Krikler DM. The Wolff-Parkinson-White and related syndromes. In: Krikler DM, Goodwin JF, eds. Cardiac arrhythmias, the modern electrophysiological approach. London: WB Saunders, 1975:144-81.

15 Rowland E, Curry P, Fox K, Krikler D. Relation between atrioventricular pathways and ventricular response during atrial fibrillation and flutter. $\mathrm{Br}$ Heart $J$ 1981;45:83-7.

16 Rahimtoola SH, Zipes DP, Akhtar M, et al. Consensus statement of the conference on the state-of-the art of electrophysiologic testing in the diagnosis and treatment of patients with cardiac arrhythmias. Circulation 1987;75(suppl III):3-11.

17 Krikler DM. Wolff-Parkinson-White syndrome: long follow-up and an Anglo-American historical note. J Am Coll Cardiol 1983;2:1216-8. 